\title{
Uso de filmes como estratégia no ensino de Bioética
}

\author{
Use of fillms as a strategy in teaching Bioethics \\ Uso de películas como estrategia en la enseñanza de la Bioética
}

Recebido: 28/04/2021 | Revisado: 05/05/2021 | Aceito: 21/05/2021 | Publicado: 08/06/2021

\author{
Davi Porfirio da Silva \\ ORCID: https://orcid.org/0000-0002-1856-4512 \\ Universidade Federal de Alagoas, Brasil \\ E-mail: daviporfirio14@hotmail.com \\ Diana Hadaça de Lima Araújo Vilela \\ ORCID: https://orcid.org/0000-0002-0042-3535 \\ Universidade Federal de Alagoas, Brasil \\ E-mail: diana.vilela@ifac.edu.br \\ Fabianny Torres de Oliveira \\ ORCID: https://orcid.org/0000-0001-6193-2002 \\ Universidade Federal de Alagoas, Brasil \\ E-mail: fabianny_torres@hotmail.com \\ Isabel Comassetto \\ ORCID: https://orcid.org/0000-0002-2389-9384 \\ Universidade Federal de Alagoas, Brasil \\ E-mail: isabelcomassetto@gmail.com \\ Regina Maria dos Santos \\ ORCID: https://orcid.org/0000-0002-2144-2997 \\ Universidade Federal de Alagoas, Brasil \\ E-mail: relpesantos@gmail.com
}

\begin{abstract}
Resumo
O objetivo desse estudo é analisar a produção cientifica sobre o uso de filmes como estratégia no ensino de bioética. Trata-se de uma revisão integrativa de literatura, com resgate de estudos a partir das bases de dados LILACS, BDENF, MEDILINE e na Biblioteca Virtual SciELO, por meio dos DeCS/MeSH Bioethics, Teaching, Education, Nursing, Instructional Film and Video, Motion Pictures e as palavras-chave Movies e Cinema. Foram selecionados oito estudos, distribuídos uniformemente nos últimos 10 anos, com texto em inglês, espanhol e português, resgatados a partir das bases de dados LILACS e MEDLINE, todos classificados no nível de evidência IV. Nesse contexto, os estudos resgatados nessa revisão apontam resultados positivos quanto ao uso de filmes no ensino de bioética, afirmando a importância da incorporação dessa estratégia nas práticas pedagógicas dos cursos de saúde. Houve massificação na concordância do uso dessa ferramenta para discussão de ideias morais e filosóficas novas ou complexas, que impactam diretamente nas questões da prática clínica no âmbito dos profissionais da saúde. Porém, a literatura ressalta que essa estratégia não deve ser usada como entretenimento despretensioso e sem compromisso.
\end{abstract}

Palavras-chave: Bioética; Ensino; Filmes; Cinema; Enfermagem.

\begin{abstract}
The objective of this study is to analyze the scientific production on the use of films as a strategy in teaching bioethics. This is an integrative literature review, with retrieval of studies from the LILACS, BDENF, MEDILINE databases and the SciELO Virtual Library, through the DeCS / MeSH Bioethics, Teaching, Education, Nursing, Instructional Film and Video, Motion Pictures and the keywords Movies and Cinema. Eight studies were selected, uniformly distributed over the last 10 years, with text in English, Spanish and Portuguese, retrieved from the LILACS and MEDLINE databases, all classified at level of evidence IV. In this context, the studies rescued in this review point to positive results regarding the use of films in the teaching of bioethics, affirming the importance of incorporating this strategy in the pedagogical practices of health courses. There was widespread agreement on the use of this tool to discuss new or complex moral and philosophical ideas, which directly impact on clinical practice issues within the scope of health professionals. However, the literature emphasizes that this strategy should not be used as unpretentious and uncompromising entertainment.
\end{abstract}

Keywords: Bioethics; Teaching; Movies; Cinema; Nursing.

\section{Resumen}

El objetivo de este estudio es analizar la producción científica sobre el uso del cine como estrategia en la enseñanza de la bioética. Se trata de una revisión bibliográfica integradora, con recuperación de estudios de las bases de datos LILACS, BDENF, MEDILINE y la Biblioteca Virtual SciELO, a través del DeCS / MeSH Bioética, Docencia, Educación, Enfermería, Cine y Video Instruccional, Motion Pictures y las palabras clave Movies y Cinema. Se 
seleccionaron ocho estudios, distribuidos uniformemente en los últimos 10 años, con texto en inglés, español y portugués, recuperados de las bases de datos LILACS y MEDLINE, todos clasificados a nivel de evidencia IV. En este contexto, los estudios rescatados en esta revisión apuntan a resultados positivos en cuanto al uso de películas en la enseñanza de la bioética, afirmando la importancia de incorporar esta estrategia en las prácticas pedagógicas de los cursos de salud. Hubo un acuerdo generalizado sobre el uso de esta herramienta para discutir ideas filosóficas y morales nuevas o complejas, que impactan directamente en cuestiones de práctica clínica dentro del ámbito de los profesionales de la salud. Sin embargo, la literatura enfatiza que esta estrategia no debe usarse como entretenimiento sin pretensiones y sin concesiones.

Palabras clave: Bioética; Enseñando; Películas; Cine; Enfermería.

\section{Introdução}

Ensinar bioética nos sistemas educacionais que formam profissionais de saúde é um desafio, pois é preciso que os estudantes exercitem a análise das suas práticas, sejam elas individuais ou coletivas, facilitando o enfrentamento de problemas e implicações éticas e morais que surgirão na vida profissional (Warmling et al., 2016). Segundo Barros et al. (2020), a ética ensinada na escola será responsável por conduzir o ser humano à condição de crítico e responsável pelos seus atos.

Por serem crescentes as preocupações e os problemas que envolvem a bioética, surgem diversos ambientes de discussão, estratégias e abordagens de estudo, sendo a arte e, sobretudo, o cinema ferramentas valorosas para representar as mudanças nas problematizações éticas na área da saúde (Antunes et al., 2016)

A arte cinematográfica é um recurso bastante utilizado no chamado "ensino da bioética", por ser um recurso pedagógico que possibilita problematizar a realidade através de reflexões e discussões (Hoffmann; Moratelli \& Finkler, 2017). Rego \& Palacios (2017) trazem em seu estudo que filmes, além de provocarem reações emocionais, como indignação e empatia, produzem um conglomerado de informações históricas que possibilitam a contextualização de conflitos morais e que, se usados em práticas organizadas, são ferramentas muito úteis aos propósitos pedagógicos.

Por possuir um caráter multidisciplinar e aliar tecnologias modernas às representações de épocas longínquas, é notório o valor estético e educativo do cinema, que se faz presente em domínios diversos da vida cotidiana, incrementando discussões necessárias ao desenvolvimento da fundamentação da bioética (González \& González-Pérez, 2019).

A literatura sobre o tema ainda se mostra limitada, o que justifica a relevância deste estudo que pretende contribuir com uma síntese do estado da produção científica, integrando estudos publicados recentemente, particularmente desde 2010, em contexto nacional e internacional, a fim de que se possa verificar a tendência do uso de tal ferramenta, verificando seus contrastes e benefícios no contexto educativo.

Desse modo, o objetivo desse estudo é analisar a produção cientifica sobre o uso de filmes como estratégia no ensino de bioética.

\section{Metodologia}

Trata-se de uma revisão Integrativa da Literatura, um estudo de síntese do conhecimento a partir da análise das evidências disponíveis, de modo sistemático e rigoroso. Desse modo, cumprindo o rigor metodológico para a elaboração de uma revisão integrativa, cumpriu-se as seis etapas: identificação do tema e seleção da questão de pesquisa; estabelecimento de critérios para inclusão e busca de estudos na literatura; definição das informações a serem extraídas dos estudos selecionados; avaliação dos estudos incluídos na revisão integrativa; interpretação dos resultados e apresentação da síntese do conhecimento (Mendes; Silveira \& Galvão; 2008; Ercole; Melo \& Alcoforado; 2014; Mendes; Silveira \& Galvão, 2019).

A questão norteadora desse estudo foi construída a partir da estratégia PICo (Problema, fenômeno de Interesse e Contexto), organizando da seguinte forma: Quais as evidências científicas disponíveis acerca do uso de filmes como estratégia no ensino de bioética? 
A seleção dos estudos foi realizada em novembro de 2020. Os critérios de inclusão adotados foram estudos publicados em português, inglês ou espanhol, no período entre 2010 e 2020, com disponibilidade na íntegra. Excluíram-se editoriais, dissertações, teses e artigos repetidos em mais de uma base de dados.

O resgate dos estudos ocorreu a partir dos descritores controlados DeCS/MeSH (Descritores em Ciências da Saúde / Medical Subject Headings): "Bioethics", "Teaching", "Education, Nursing", "Instructional Film and Video" e "Motion Pictures", combinados por meio operador booleano AND. Além disso, foi necessário o uso dos descritores não controlados "Movies" e "Cinema". As bases de dados consultadas foram: MEDLINE (Medical Literature Analysis and Retrieval System Online), LILACS (Literatura Latino-Americana e do Caribe em Ciências da Saúde), BDENF (Base de Dados de Enfermagem), por meio da Biblioteca Virtual em Saúde (BVS), e a biblioteca virtual SciELO.

A descrição da busca e seleção dos estudos foi realizada a partir das recomendações PRISMA (Liberati et al., 2009), conforme a Figura 1.

Figura 1. Fluxograma de busca e seleção dos estudos incluídos na revisão baseadas nas recomendações PRISMA.
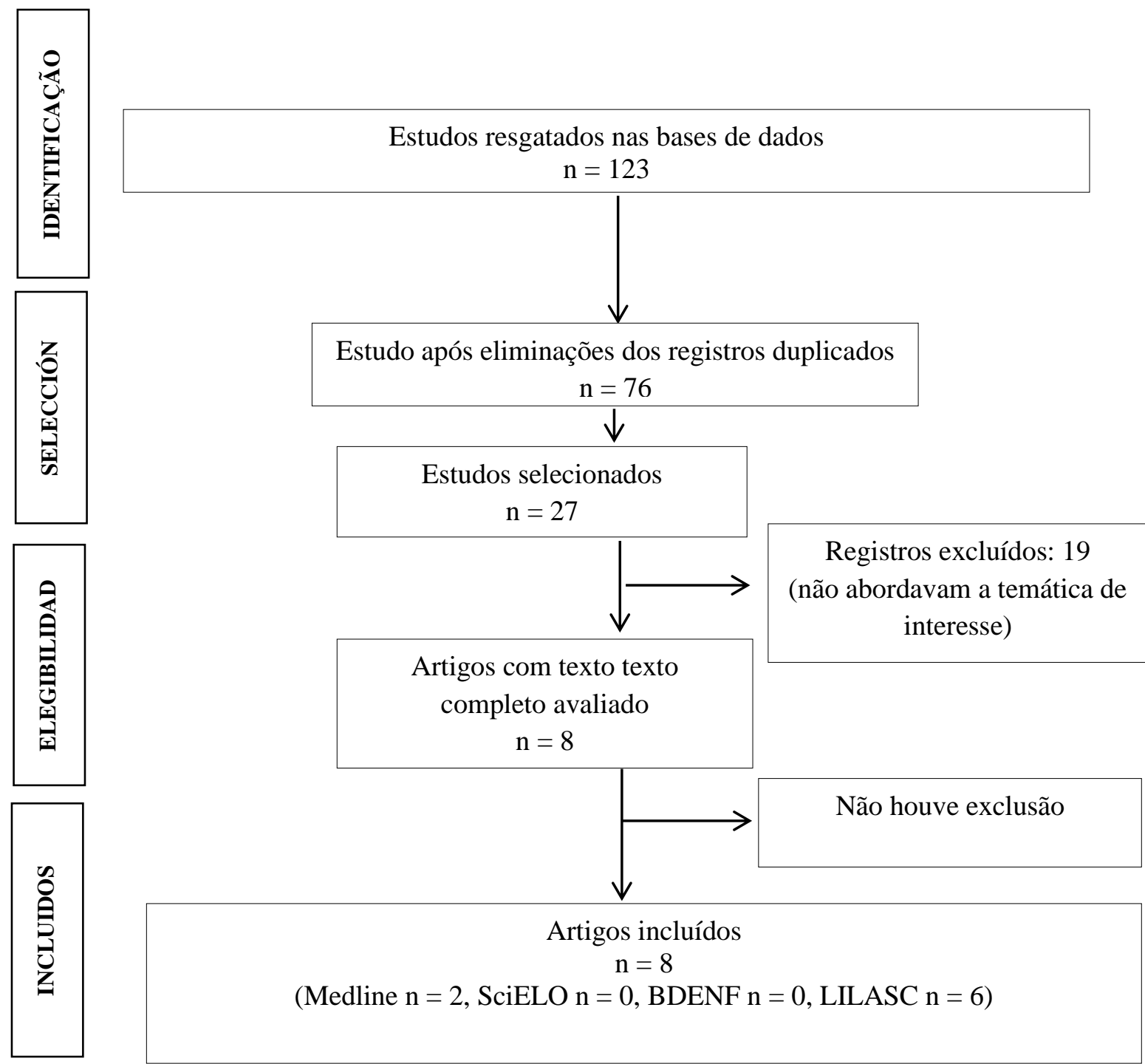

Fonte: Autores (2021). 
A coleta de dados dos artigos selecionados foi realizada por meio de instrumento elaborado pelos autores, baseado em Ursi (2005), contendo itens relacionados à identificação do estudo, caracterização da publicação, objetivo, metodologia, principais resultados. Os estudos selecionados foram avaliados criteriosamente e classificados quanto ao nível de evidência. A síntese da revisão ocorreu de forma qualitativa, discutindo-se avanços e lacunas no conhecimento.

\section{Resultados e Discussão}

Foram selecionados oito estudos por meio das buscas nos bancos de dados. As publicações distribuíram-se uniformemente nos últimos 10 anos, sendo regatados artigos publicados em inglês, espanhol e português, a partir das bases de dados LILACS e MEDLINE.

Ao analisar os aspectos metodológicos dos estudos incluídos nessa revisão, considerando a força das evidências resgatadas, conforme Souza, Silva e Carvalho (2010), todos os estudos foram classificados no nível IV, que compreendem evidências de estudos descritivos (não-experimentais) ou com abordagem qualitativa.

A síntese dos estudos selecionados, quantos às características de publicação, objetivo, metodologia e principais resultados está disposta no Quadro 1. 
Quadro 1 - Síntese dos estudos avaliados conforme autores, título do artigo, principais resultados, autores, síntese e nível das evidências.

\begin{tabular}{|c|c|c|c|c|c|}
\hline Autores & Título & $\begin{array}{l}\text { Informação de } \\
\text { publicação }\end{array}$ & Método & Principais resultados & $\begin{array}{l}\text { Nível de } \\
\text { Evidência }\end{array}$ \\
\hline $\begin{array}{l}\text { Alerm-González, } \\
\text { Alina J.; González- } \\
\text { Pérez, Ubaldo. }\end{array}$ & $\begin{array}{l}\text { Propuesta del cine de } \\
\text { ciencia ficción para } \\
\text { educar en Bioética }\end{array}$ & $\begin{array}{l}\text { Pers. bioet; 23(1): } \\
\text { 14-33, jun. } 2019 . \\
\text { Base de dados: } \\
\text { Lilacs }\end{array}$ & $\begin{array}{l}\text { Estudo } \\
\text { Reflexão }\end{array}$ & $\begin{array}{l}\text { Considerando as potencialidades do uso de filmes } \\
\text { para educar estudantes de medicina em bioética, } \\
\text { os autores apresentam alguns filmes que tratam } \\
\text { de temas sensíveis sob o olhar da bioética. }\end{array}$ & IV \\
\hline $\begin{array}{l}\text { Ike, Chiedozie G; } \\
\text { Anderson, Nancy }\end{array}$ & $\begin{array}{l}\text { A proposal for } \\
\text { teaching bioethics in } \\
\text { high schools using } \\
\text { appropriate visual } \\
\text { education tools }\end{array}$ & $\begin{array}{l}\text { Philos } r \text { Ethics } \\
\text { Humanit Med; } 13 \\
\text { (1): 11, } 20180720 . \\
\text { Base de dados: } \\
\text { Medline }\end{array}$ & Estudo Revisão & $\begin{array}{l}\text { Os autores evidenciam que o ensino de bioética } \\
\text { com ferramentas de educação visual são úteis } \\
\text { para o ensino de ética médica e em pesquisa, } \\
\text { melhorando a compreensão de questões morais e } \\
\text { legais complexas. }\end{array}$ & IV \\
\hline $\begin{array}{l}\text { Pereira Rates, Camila } \\
\text { Maria; Maciel Silva, } \\
\text { Larriny; Moura } \\
\text { Pereira, Lívia; Reis } \\
\text { Pessalacia, Juliana } \\
\text { Dias. }\end{array}$ & $\begin{array}{l}\text { The use of films as a } \\
\text { teaching tool for the } \\
\text { teaching-learning } \\
\text { process in bioethics }\end{array}$ & $\begin{array}{lr}\text { Invest } & \text { Educ } \\
\text { Enferm; } 32(3): \\
\text { 421-9, 2014. } \\
\text { Base de dados: } \\
\text { Medline }\end{array}$ & $\begin{array}{l}\text { Estuco com } \\
\text { abordagem } \\
\text { qualitativa }\end{array}$ & $\begin{array}{l}\text { Esse estudo aponta experiências positivas no uso } \\
\text { de filmes no ensino de bioética. Estudantes da } \\
\text { saúde entrevistados relataram mudanças na } \\
\text { postura ética após discussões de filmes exibidos. }\end{array}$ & IV \\
\hline $\begin{array}{lr}\text { Cezar, } & \text { Pedro } \\
\text { Henrique } & \text { Netto; } \\
\text { Gomes, } & \text { Andréia } \\
\text { Patrícia; } & \text { Siqueira- } \\
\text { Batista, Rodrigo. }\end{array}$ & $\begin{array}{l}\text { O cinema e a } \\
\text { educação bioética no } \\
\text { curso de graduação } \\
\text { em Medicina }\end{array}$ & $\begin{array}{l}\text { Rev. bras. educ. } \\
\text { méd; 35(1): 93- } \\
\text { 101, jan.-mar. } \\
\text { 2011. tab } \\
\text { Base de dados: } \\
\text { Lilacs }\end{array}$ & $\begin{array}{l}\text { Estudo } \\
\text { reflexão }\end{array}$ & $\begin{array}{l}\text { Para os autores, o cinema emerge como } \\
\text { ferramenta eficaz no ensino da bioética, porque } \\
\text { recria, nas telas, situações bem próximas da } \\
\text { realidade. Estas podem provocar emoções e } \\
\text { pensamentos capazes de deflagrar uma reflexão } \\
\text { sobre questões morais, habituando o aprendiz a } \\
\text { utilizar o arco prática-teoria-prática na resolução } \\
\text { de seus problemas. }\end{array}$ & IV \\
\hline $\begin{array}{l}\text { Morales Guzmán- } \\
\text { Barrón, Rosanna. }\end{array}$ & $\begin{array}{lr}\text { El cine } & \text { como } \\
\text { herramienta } & \text { de } \\
\text { motivación } & y \\
\text { educación del médico } \\
\text { en bioética }\end{array}$ & $\begin{array}{lr}\text { Rev. } & \text { Soc. } \\
\text { Med. Peru. } \\
\text { 24(2): } \text { Interna; } \\
\text { abr.-jun. } 2011.107, \\
\text { Base de dados: } \\
\text { Lilacs }\end{array}$ & $\begin{array}{l}\text { Estudo } \\
\text { revisão }\end{array}$ & $\begin{array}{l}\text { Os autores apontam que os filmes podem ser uma } \\
\text { ferramenta útil para ensino da bioética. A } \\
\text { exibição de filmes no ensino dessa disciplina } \\
\text { promove um espaço individualizado para } \\
\text { reflexão. }\end{array}$ & IV \\
\hline $\begin{array}{l}\text { Muniz, Ianna; Lins, } \\
\text { Liliane; } \quad \text { Menezes, }\end{array}$ & $\begin{array}{l}\text { Uso de documentário } \\
\text { no curso de medicina }\end{array}$ & $\begin{array}{l}\text { Rev. bioét. (Impr.); } \\
26(4): \quad 606-616,\end{array}$ & $\begin{array}{l}\text { Estudo } \\
\text { descritivo, com }\end{array}$ & $\begin{array}{l}\text { Estudo aponta que a exibição do filme "À } \\
\text { margem do corpo" serviu como instrumento de }\end{array}$ & IV \\
\hline
\end{tabular}


Research, Society and Development, v. 10, n. 6, e49710615559, 2021

(CC BY 4.0) | ISSN 2525-3409 | DOI: http://dx.doi.org/10.33448/rsd-v10i6.15559

\begin{tabular}{|c|c|c|c|c|c|}
\hline Marta Silva & $\begin{array}{ll}\text { e a reflexão sobre } \\
\text { temas } & \text { éticos } \\
\text { associados ao aborto }\end{array}$ & $\begin{array}{l}\text { out.-dez. } 2018 . \\
\text { Base de dados: } \\
\text { Lilacs }\end{array}$ & $\begin{array}{l}\text { método } \\
\text { qualitativo }\end{array}$ & $\begin{array}{l}\text { ensino-aprendizagem sobre aspectos éticos e } \\
\text { bioéticos relacionados à vulnerabilidade e } \\
\text { autonomia da mulher, direito médico à objeção de } \\
\text { consciência e negligência na atenção à saúde, } \\
\text { assim como os efeitos negativos de conduta } \\
\text { inadequada na assistência na interrupção da } \\
\text { gestação. }\end{array}$ & \\
\hline $\begin{array}{l}\text { Aleksandrova- } \\
\text { Yankulovska, Silviya }\end{array}$ & $\begin{array}{l}\text { An innovative } \\
\text { approach to teaching } \\
\text { bioethics } \\
\text { management in } \\
\text { healthcare }\end{array}$ & $\begin{array}{l}\text { Nurs Ethics; 23(2): } \\
\text { 167-75, 2016 Mar. } \\
\text { Base de dados: } \\
\text { Lilacs }\end{array}$ & $\begin{array}{l}\text { Estudo } \\
\text { qualitativo }\end{array}$ & $\begin{array}{l}\text { Para os autores a introdução do cinema no ensino } \\
\text { de bioética, realizado por meio da exibição de } \\
\text { dois filmes, teve o potencial de fornecer } \\
\text { ilustrações vívidas de questões bioéticas e de } \\
\text { contribuir para a exploração de teses e } \\
\text { argumentos específicos. }\end{array}$ & IV \\
\hline $\begin{array}{lr}\text { Dantas, } & \text { Anielle } \\
\text { Avelina; } & \text { Martins, } \\
\text { Carlos } & \text { Henrique; } \\
\text { Militão, } & \text { Maria } \\
\text { Socorro Ramos. }\end{array}$ & $\begin{array}{l}\text { O cinema como } \\
\text { instrumento cidático } \\
\text { para a abordagem de } \\
\text { problemas bioéticos: } \\
\text { uma reflexão sobre a } \\
\text { eutanásia }\end{array}$ & $\begin{array}{l}\text { Rev. bras. educ. } \\
\text { méd; 35(1): 69-76, } \\
\text { jan.-mar. } 2011 . \\
\text { Base de dados: } \\
\text { Lilacs }\end{array}$ & $\begin{array}{l}\text { Estudo } \\
\text { qualitativo }\end{array}$ & $\begin{array}{l}\text { Conforme os autores a exibição de um filme } \\
\text { seguida da discussão de cenas pode ser utilizada } \\
\text { como instrumento pedagógico significante para } \\
\text { atingir objetivos educacionais humanísticos dos } \\
\text { currículos dos cursos de Medicina. }\end{array}$ & IV \\
\hline
\end{tabular}

Fonte: Autores (2021). 
Devido às transformações advindas do desenvolvimento científico, tecnológico e social das últimas décadas, o mercado de trabalho demanda por profissionais com capacidades e competências indispensáveis as modificações tecnológicas, conceituais e de valor do meio sociocultural (Neves Júnior; Araújo \& Rego, 2016), bem como a sociedade moderna exige um profissional de saúde com capacidade de ação e resolutividade baseadas na ética (Pissaia, 2019).

Porém, mesmo diante deste cenário, nota-se que o ensino da bioética na modernidade ainda é uma fragilidade no que diz respeito à atenção dispensada pelos profissionais das ciências da saúde, que estar presente devido ao pouco tempo de existência deste conceito e o desafio que é sensibilizar os acadêmicos no desenvolvimento de valores morais e atitudes respeitosas ao lidar com os impasses decorrentes de novas tecnologias e o pluralismo cultural. Diante disto, as Diretrizes Curriculares de cursos de graduação e pós-graduação recomendam o uso de estratégias que desenvolvam o conhecimento integral, ético e humanizado (Biondo; Rosa; Ferraz \& Yarid, 2018).

É comum que, ao ensinar bioética na área da saúde, sempre existirão especificidades a serem consideradas de acordo com a sociedade atual, o que gera uma preocupação no que diz respeito às práticas pedagógicas que serão utilizadas. No ensino superior brasileiro, em níveis de graduação e pós-graduação, em cursos como enfermagem, medicina e ciências biológicas, a bioética é inserida a fim de conceber aos futuros profissionais habilidade técnica aliada a comportamentos humanitários na resolução de dilemas éticos contemporâneos (Fischer; Cunha; Roth; Martins, 2017).

Nunes (2017) afirma que não existe uma única tradição pedagógica que dê conta de toda a interdisciplinaridade da bioética, surgindo diversas abordagens pedagógicas, como fóruns de internet, blogs e situações-problema e que a utilização de produções cinematográficas desponta como uma das estratégias mais utilizadas para que se seja possível a compreensão da bioética em sua concepção, implementação, monitorização e avaliação.

Os estudos resgatados nessa revisão apontam resultados positivos quanto ao uso de filmes no ensino da bioética. Dantas et al. (2011), ao analisar o ensino da bioética no contexto da eutanásia, apontaram que a exibição de um filme seguida da discussão de cenas pode ser utilizada como instrumento pedagógico significante para atingir objetivos educacionais humanísticos dos currículos dos cursos de medicina. De modo semelhante, Aleksandrova-Yankulovska (2016), por meio de um estudo qualitativo sobre ensino da bioética, evidenciou que a introdução do cinema no ensino de bioética realizado por meio da exibição de dois filmes teve o potencial de fornecer ilustrações vívidas de questões bioéticas e de contribuir para a exploração de teses e argumentos específicos.

Alerm-González e González-Perez (2019) ao apontarem em seus estudos alguns filmes que abordam temas sensíveis à questões da bioética, como Planeta dos Macacos e o Homem Bicentenário, afirmam que estes são capazes de despertar emoções e sentimentos, sendo recursos indispensáveis para uma reconstrução afetiva, tendo o espectador à possibilidade de discutir abertamente, fazer comparações com situações vivenciadas por ele e compartilhá-las com outras pessoas, desenvolvendo assim suas habilidades para enfrentar questões éticas e morais. Segundo Fischer et al. (2017) muitos estudantes detentores de pouco conhecimento acerca de temas atuais e polêmicos debatidos na mídia, apresentam maior interesse e envolvimento para desenvolver pesquisas e aprofundar seus conhecimentos, após assistirem algum filme que aborde a temática em questão.

Ao pensar nas ferramentas visuais como instrumento de ensino da bioética é importante considerar especificidades do público alvo como: idade, raça, gênero, crenças, nível de educação e orientação sexual. A seleção do material (filme) a ser utilizado também precisa ser previamente pensado para que não traga ideias utópicas, distópicas ou estereótipos sociais. O conteúdo precisa ser o mais realista e concreto possível, afim de que se alcance o objetivo proposto com o uso da ferramenta, que é aumentar a compreensão de questões morais e jurídicas na saúde e na pesquisa (Ike \& Anderson, 2018).

A formação nos diversos cursos da saúde atualmente se volta, conforme determinação das Diretrizes Curriculares Nacionais da Educação, para o desenvolvimento de uma postura ética e humanizada que deve ser precocemente estimulada na 
formação (Cezar; Gomes \& Batista, 2011). Segundo Pereira et al (2014), ao abordar alunos de graduação da saúde sobre o uso de filmes no processo ensino-aprendizagem em bioética, a partir do referencial de Bardin, estudante que vivenciaram essa experiência referiram em suas respostas a importância da visão do problema bioético para reflexão e decisão na prática profissional. Mais ainda, houve relatos de mudanças na postura ética a partir das discussões de filmes exibidos, apontando esta como uma potente ferramenta.

Temas que envolvem os mais diversos dilemas éticos podem ser abordados utilizando-se a ferramenta de filmes. Em nossas buscas os temas eutanásia e aborto foram diretamente trabalhados em dois artigos distintos. A temática aborto foi abordada utilizando a exibição do documentário A margem do corpo, com posterior discussão de cenas selecionadas. No questionário respondido pelos participantes, além do tema central aborto, surgiram outras três temáticas importantes para o ensino da bioética: 1) vulnerabilidade e redução da autonomia; 2) objeção de consciência e 3) desumanização e negligência na assistência à saúde (Muniz; Lins \& Menezes, 2018).

Apesar de apenas dois temas importantes para a bioética terem sido diretamente tratados nos estudos encontrados, todos corroboram ao trazer a importância e eficácia do uso de tecnologias leves, principalmente da indústria cinematográfica, na formação ética dos profissionais de saúde. Vários dilemas e conceitos éticos foram apontados por um grupo de estudantes da área de gestão em saúde, após assistirem dois filmes com abordagem ética, o que aponta um indicador positivo de maior sensibilização ética (Aleksandrova-Yankulovska, 2016).

\section{Considerações Finais}

Os estudos regatados nessa revisão evidenciam o uso de filmes como uma estratégia de ensino útil à bioética. Houve massificação na concordância do uso dessa ferramenta para discussão de ideias morais e filosóficas novas ou complexas, que impactam diretamente nas questões da prática clínica no âmbito dos profissionais da saúde. Porém, também foi apontada, para o alcance do objetivo proposto, a importância do uso de filmes em consonância com os temas abordados e não como entretenimento despretensioso e sem compromisso. Salienta-se a necessidade de novos estudos para fortalecer o corpo de conhecimento na área.

\section{Referências}

Aleksandrova-Yankulovska, S. (2016). An innovative approach to teaching bioethics in management of healthcare. Nurs Ethics; 23(2), 167-75. https://doi.org/10.1177/0969733014558967.

Alerm-González, A. J. \& González-Pérez, U. (2019). Propuesta del cine de ciencia ficción para educar en Bioética. Pers. Bioet, 23(1), 14-33. https://doi.org/10.5294/pebi.2019.23.1.2.

Antunes, M. S. B. et al. (2016). A arte cinematográfica e a bioética: reflexão sobre a eutanásia. Revista Uningá, 49 (1) http://34.233.57.254/index.php/uninga/article/view/1304.

Barros, L. B. L. et al. (2020). Ética, Legislação e Educação: o tripé ambiental. Research, Society And Development, 10 (9), 1-20. http://dx.doi.org/10.33448/rsd-v9i10.8601.

Biondo, C. S. et al. (2018). Perspectivas do conhecimento da bioética pelos acadêmicos de saúde para atuação profissional. Enfermería Actual En Costa Rica, 35, 1-12, http://dx.doi.org/10.15517/revenf.v0i35.30014.

Cezar, P. H. N., Gomes, A. P. \& Siqueira-Batista, R. (2011). O cinema e a educação bioética no curso de graduação em Medicina. Rev. bras. educ. méd, 35 (1), 93-101. https://doi.org/10.1590/S0100-55022011000100013

Dantas, A. A., Martins, C. H. \& Militão, M.S.R. (2011). O cinema como instrumento didático para a abordagem de problemas bioéticos: uma reflexão sobre a eutanásia. Rev. bras. educ. méd, 35 (1), 69-76. https://doi.org/10.1590/S0100-55022011000100010

Ercole, F.F, Melo, L.S. \& Alcoforado, C. L. G. C. Revisão integrativa versus revisão sistemática (2014). Rev. REME, 18 (1), 1-2. https://doi.org/10.5935/14152762.20140001.

Fischer, M. L. et al. (2017). Caminho do Diálogo: uma experiência bioética no ensino fundamental. Revista Bioética, 25, (1), 89-100. http://dx.doi.org/10.1590/1983-80422017251170. 
Research, Society and Development, v. 10, n. 6, e49710615559, 2021

(CC BY 4.0) | ISSN 2525-3409 | DOI: http://dx.doi.org/10.33448/rsd-v10i6.15559

Guzmán-Barrón, R. M. (2011). El cine como herramienta de motivación y educación del médico en bioética. Rev. Soc. Peru. Med. Interna, 24 (2), 103-107. https://doi.org/10.36393/spmi.v24i2.454.

Hoffmann, J. N., Moratelli, L. B.; Finkler, M. (2017). Educação permanente em saúde: uma experiência do projeto bioética pelas lentes do cinema. Extensio: Revista Eletrônica de Extensão, 26 (14), 97-106, 2017. http://dx.doi.org/10.5007/18070221.2017v14n26p97.

Ike, C. G., Anderson, N. (2018). A proposal for teaching bioethics in high schools using appropriate visual education tools. Philos Ethics Humanit Med, 13 (1): 11. http://dx.doi.org/10.1186/s13010-018-0064-1.

Liberati, A. et al. (2009). The PRISMA Statement for Reporting Systematic Reviews and Meta-Analyses of Studies That Evaluate Health Care Interventions: Explanation and Elaboration. Journal of Clinical Epidemiology, 62 (10), e1-34. https://doi.org/10.1016/j.jclinepi.2009.06.006.

Mendes, K. D. S., Silveira, R. C. C. P., Galvão, C. M. (2008). Revisão integrativa: método de pesquisa para a incorporação de evidências na saúde e na enfermagem. Texto Contexto Enferm, 17 (4), 758-64. https://doi.org/10.1590/S0104-07072008000400018.

Mendes, K. D. S., Silveira, R. C. C. P., Galvão, C.M. (2018). Uso de gerenciador de referências bibliográficas na seleção dos estudos primários em revisão integrativa. Texto contexto enferm, 28, e20170204. https://doi.org/10.1590/1980-265x-tce-2017-0204.

Muniz, I., Lins, L. \& Menezes, M. S. (2018). Uso de documentário no curso de medicina e a reflexão sobre temas éticos associados ao aborto. Rev. bioét., 26(4): 606-616. http://dx.doi.org/10.1590/1983-80422018264279.

Neves Júnior, W. A., Araújo, L. S. \& Rego, S.(2016). Ensino de bioética nas faculdades de medicina no Brasil. Rev. Bioét, 24 (1), $98-107$. http://dx.doi.org/10.1590/1983-80422016241111.

Nunes, L. (2017). Do ensino da bioética e as escolhas temáticas dos estudantes. Rev. Bioét, 25 (3), 512-526. http://dx.doi.org/10.1590/1983-80422017253208.

Pissaia, L. F. (2019). Ética profissional e seu ensino em cursos de graduação na área da saúde. Research, Society And Development, 8 (1), 1-14. https://dx.doi.org/10.33448/rsd-v8i1.627.

Rates, C. M. P., Larriny, M. C., Lívia, M. P. \& Pessalacia, J. D. R. (2014). The use of films as a teaching tool for the teaching-learning process in bioethics. Invest Educ Enferm, 32 (3), 421-9. https://dx.doi.org/10.17533/udea.iee.v32n3a07.

Rego, S., \& Palacios, M. (2017). Contribuições para planejamento e avaliação do ensino da bioética. Rev. Bioét, 25(2): 234-243. http://dx.doi.org/10.1590/1983-80422017252183.

Souza, M. T., Silva, M. D. \& Carvalho, R. (2010). Revisão integrativa: o que é e como fazer. Einstein. 8 (1), 102-106. https://doi.org/10.1590/s167945082010rw1134.

Ursi, E. S. Prevenção de lesões de pele no perioperatório: revisão integrativa da literatura. [dissertation]. Ribeirão Preto: Universidade de São Paulo, Escola de Enfermagem de Ribeirão Preto; 2005.

Warmling, C. M. et al. (2016). Ensino da bioética: avaliação de um objeto virtual de aprendizagem. Revista Bioética, 24(3), 503-514. http://dx.doi.org/10.1590/1983-80422016243150. 